\title{
Obtención de Biodiesel utilizando fenómenos catalíticos a partir de la semilla de la Jatropha curcas. Una estrategia para el desarrollo de competencias investigativas
}

\author{
Biodiesel prduction using catalytic phenomenon through of the Jatropha curcas \\ seed. A strategy to develop investigative competitions
}

Katherine Montaña, Paola Moreno, Angélica Torres y Rodrigo Rodríguez

${ }^{1}$ Estudiantes de Décimo Semestre de Licenciatura en Química y Profesor del Departamento de Química. Universidad Pedagógica Nacional. Bogotá. Grupo de investigación Didáctica y sus ciencias.dqu578_kamontana@pedagogica.edu.co, dqu679 pmoreno@pedagogica.edu.co dqu602_atorres@pedagogica.edu.co, rrodriguez@pedagogica.edu.co

\section{Resumen}

En aras de forjar una propuesta cognitiva que promueva en el estudiante y en la comunidad cientúfica la comprensión, construcción y asimilación de conceptos propios de un área de la química como lo es la catálisis, sustentada a través de la obtención de biodiesel a partir de la semilla de la Jatropha Curcas, así como la ejecución de habilidades cient́ficas, se propone la implementación de una estrategia pedagógica y didáctica centrada en las competencias investigativas orientadas desde la resolución problemas, con el fin de brindar a la comunidad científica las herramientas para que definan problemáticas ambientales, sociales y económicas, y propongan posibles soluciones para las mismas, manifestando de este modo la capacidad para comunicar adecuadamente los resultados en el campo investigativo.

Palabras clave

Competencias Investigativas, Enseñanza - Aprendizaje, Estrategia Pedagógica - Didácica, Habilidades Científicas, Resolución de Problemas

\begin{abstract}
A proposal cognitive that promotes in the student and in the scientific community the understanding, construction and assimilation of concepts characteristic of an area of the chemistry as it is the catalysis, sustained through the biodiesel obtaining from the Jatropha Curcas's seed, as well as the execution of scientific abilities, we propose the implementation of a pedagogic and didactics strategy, that it centered in the investigative competitions. They guided from the resolution problems, to give some tools to the scientific community, so that they define environmental, social and economic problems, and they propose possible solutions for the same ones, manifesting the capacity to communicate the results appropriately in the investigative field.
\end{abstract}

Key words

Investigative competitions, Teaching - Learning, Pedagogic - Didactic Strategy, Scientific Skills, Resolution problems.

\section{Introducción}


Se presenta una propuesta metodológica de enseñanza aprendizaje por competencias investigativas dirigida fundamentalmente en la resolución de problemas, que integra conceptos relacionados con los fenómenos catalíticos implicados en la obtención de un biocombustible, como una alternativa de solución a los problemas ambientales, sociales y económicos, tales como la emisión de gases de efecto invernadero, el uso de energías fósiles, el incremento en el precio de los alimentos, el establecimiento de monocultivos así como la utilización de agroquímicos nocivos para el medio ambiente y la destrucción de ecosistemas naturales, entre otros. Este enfoque posibilita formar profesionales idóneos capaces de promover proyectos y programas de investigación con el fin de aportar soluciones a los problemas de relevancia social. La estrategia metodológica se apoya en la elaboración e implementación de un modulo didáctico de soporte con el ánimo de fortalecer las competencias investigativas, tanto en un grupo de estudiantes como en los investigadores, a través de los trabajos prácticos de laboratorio, para que reconozcan y valoren críticamente el impacto social y ambiental de los conocimientos científicos.

\section{Referentes}

El concepto de competencias llego a la educación desde el campo del lenguaje, a partir de la competencia lingüística y de la competencia comunicativa, las auales apuntan a asumir el estudio de la lengua y de la comunicación humana más allá de la transmisión de reglas y memorización del significado de las palabras. Los aportes de la lingǘstica, junto con las influencias de la teoría del procesamiento de la información, las inteligencias múltiples y las competencias laborales, llevaron a introducir el término en otras áreas del curríaulo diferentes al área del lenguaje. De este modo se consolida poco a poco el concepto de competencias (competencias comunicativas, competencias matemáticas, competencias sociales, competencias en ciencias naturales, competencias investigativas, etc.). Las competencias en la educación han sido influenciadas por una serie de factores extemos, tales como, competencias empresariales, la globalización y la internacionalización de la economía (Tobón, 2004).

De esta forma, este concepto puede ser asumido como un saber hacer razonado para ser referente a la incertidumbre; manejo de la incertidumbre en un mundo cambiante en lo social, político y lo laboral dentro de una sociedad globalizada y en continuo cambio (Bacarat y Graziano, 2002).

Las competencias investigativas, pretenden formar profesionales con amplios conocimientos y destrezas para emprender proyectos y programas de investigación de modo que pueden abordar y responder adecuadamente al estudio de problemas de relevancia social del contexto. El desarrollo de estas competencias pretende estructurar un pensamiento crítico, sistémico, abierto, reflexivo y creativo (Mendivelso, 2006). Con llevaría esto el desarrollo de ciertas habilidades básicas para la comprensión de un fenómeno que, finalmente, es el objetivo de un proceso investigativo. Estas serian: problematizar la realidad (comunidad de indagación), registrar, procesar y recuperar información para aprender a: analizar problemas; hacer inferencias (anticipación conjetural creativa); relacionar (aprendizaje por transferencia analógica); plantear y resolver problemas; elaborar conceptos y proposiciones; redactar informes; construir herramientas para llevar a cabo procesos de observación, entrevistas 0 análisis de documentos; manejar estadísticas básicas; deducir e interpretar resultados (Mendivelso, 2006).

Un componente fundamental de las competencias es la resolución de problemas. Resolver un problema no es simplemente aplicar un algoritmo lógico, realizar las operaciones establecidas y llegar a un resultado. Esta es una visión simple de este campo. Tampoco la resolución de problemas depende del grado de aprendizaje de las nociones, conceptos y categorías de una determinada disciplina, sino también de la forma como sean significados, comprendidos y abordados en un contexto. 
Por ello, la formación en problemas

Se revela como la mas fecunda para el desarrollo de las competencias, no solo por que involucra y deslinda la diversidad de problemáticas (...) sino por que en su "núcleo fuerte" asume la cognición humana como una construcción y una práctica social, relacionada con la forma como nosotros actuamos y nos relacionamos en el mundo según las representaciones, estrategias y habilidades que tenemos en un momento dado (Gómez, 2001)

En la resolución de problemas desde las competencias es preciso realizar las siguientes acciones: (1) Comprender el problema en un contexto disaiplinar, social y económico; (2) Establecer varias estrategias de solución, donde se tenga en cuenta lo imprevisto y la incertidumbre; (3) considerar las consecuencias del problema y los efectos de la solución dentro del conjunto del sistema, y (4) aprender del problema para asumir y resolver problemas similares en el futuro (Tobón, 2008).

La resolución de problemas es una estrategia didáctica que permite abordar los contenidos de curriculum desde una perspectiva interdisciplinar. Acercase, por tanto, a la resolución de un problema o situación problemática, por parte de un sujeto, supone poner a la disposición del mismo todos los recursos (intelectuales, procedimentales, instrumentales, emocionales, etc.) que conforman su bagaje formativo, hacia la búsqueda de una solución única y singular al problema planteado. La solución propuesta es un exponente del conocimiento y uso de herramientas intelectuales que el resolutor domina.

En sus onígenes, la utilización del término estuvo exclusivamente ligada a la enseñanza de las matemáticas; sin embargo, en la actualidad constituye una técnica, de enorme interés cientíico y social, de carácter interdisciplinar, caracterizada por una variedad de significados. En la sociedad de conocimiento, la necesidad de conseguir hábitos de razonamientos rigurosos y críticos, en un mundo cada vez más tecnificado, dotan al resolutor de problemas de capacidad para dirigir su desarrollo intelectual, personal y social, de una manera autónoma e independiente. Según la literatura (García, 2001, González, 2000; Adajian, 1997) la resolución de problemas se ha definido como un lugar para desarrollar un pensamiento independiente, como espacio para dotar de significados a los conceptos, a través del trabajo de producción que el resolutor desarrolla en el aurso del proceso, como henramienta diferenciada, en función del contexto que demanda la solución del problema, como heuństica que permite acercarse a cualquier ámbito educativo y contexto de interacción o como plataforma teórica y metodológica cayo objetivo es estudiar el origen y desarrollo de los procesos de representación en el niño.

Según Mata, Rodríguez y Bolívar (2004), es importante en este campo la definición o conceptualización de que es un problema. El contenido del término problema viene determinado, la mayoría de las veces, a partir de la actividad que implica más que por la forma en que se propone. En este sentido, una de las diferencias mas ampliamente utilizadas es la que distingue entre ejercicio y problema. El término "ejercicio" implica que el resolutor no conoce el procedimiento que le lleva con certeza a la posible solución. Se considera un problema como una situación que representa dificultades, para las aules no hay soluciones evidentes. En el intento de diferenciar ejercicio y problema o de establecer distintos tipos de problemas, se sigue situando la resolución de problemas dentro de dos perspectivas daras:

a) Como justificación practica de conocimientos y técnicas previamente estudiadas. Desde esta perspectiva la resolución de problemas tiene sentido como estrategia que facilita el paso desde el conocimiento a su utilización practica. Para ello, sería necesario, en primer lugar, conocer los contenidos y las técnicas operatorias, antes de su utilización. En el plano didáctico, se trata de 
buscar un determinado número de problemas, adecuados al nivel de conocimientos y lenguaje de los alumnos que les facilite esa "aplicación práctica" de los conocimientos adquiridos.

b) A partir de un contexto enunciado bien planteado. Este significado sobre la resolución de problemas aparece a partir de los años ochenta. Autores como Carpenter y Moser (1983) señalan, dentro de la enseñanza de las matemáticas, que los problemas podrían ser un elemento motivador para el estudio y comprensión de determinados conceptos matemáticos. Según ellos, los problemas verbales podrían ser utilizados como elemento base para el desarrollo de los conceptos de adición y sustracción, antes induso que el aprendizaje de las habilidades de cálaulo, que podrían surgir a partir de aquellos. La resolución de problemas no es, en este caso, una estrategia que suponga necesariamente conocimientos sobre un determinado tema, relacionado con los problemas, sino más bien un contexto, dentro del que se desarrolla la actividad matemática.

\section{Situación problemática}

Es de vital importancia tanto a nivel disciplinar como pedagógico contribuir y participar en la generación de nuevas altemativas, con el ánimo de reducir el impacto que producen los problemas ambientales, sociales y económicos que aquejan a nuestra sociedad, puesto que constituye una prioridad y una oportunidad para el país, debido a que existen una serie de limitantes o retos tecnológicos, regulatorios, económicos y ambientales que pueden afectar eslabones críticos de la cadena productiva del biocombustible y, que de no ser superados pueden conducir a su fracaso.

Se propone la producción de biodiesel, utilizando como materia prima la semilla de la Jatropha Curcas a partir de fenómenos catalíticos, como un precedente para desarrollar competencias investigativas en el campo pedagógico, en el campo social y en el campo disciplinar, valiéndonos de la resolución de problemas como una estrategia cognitiva que permita una mayor comprensión de dichos fenómenos a partir de los trabajos prácticos de laboratorio. Partiendo de lo dicho anteriormente se plantea la siguiente pregunta orientadora ¿Cómo la obtención de un biocombustible permite construir una estrategia pedagógica $y$ didáctica posibilitadora de Competencias Investigativas?

\section{Propuesta metodológica}

Cabe adarar que el presente trabajo surge como apoyo a la investigación que se está realizando como trabajo de grado.

Esta investigación tiene como objetivo elaborar e implementar un modulo pedagógico y didáctico, dirigido a un grupo de estudiantes de Licenciatura en química afines con conocimientos generales de fisicoquímica, con el fin de brindar una herramienta pedagógica basada en competencias investigativas a través de la resolución de problemas, generando en ellos una mayor comprensión de los fenómenos catalíticos.

Uno de los lineamientos de los que parte esta investigación se sustenta desde como la resolución de problemas es una estrategia para el desarrollo de competencias investigativas que posibilita la reestructuración de conceptos y métodos de modo similar a como lo hacen los grupos de investigación científica, con el fin de aproximarse a diversos procesos generados por la ciencia y que inciden en su contexto social.

En el transcurso de la investigación se realizan una serie de actividades, entendidas estas como guías, talleres, prácticas de laboratorio, lecturas, etc., basadas en la resolución de problemas, para la elaboración del modulo. Inicialmente se caracterizarán las concepciones altemativas de los 
estudiantes, se determinarán sus conocimientos acerca de los fenómenos catalíticos, así como las generalidades de los metales implicados en la obtención del biocombustible (propiedades físicas y químicas, átomo, tipo de enlace que forma, minerales y distribución en la naturaleza; también se deben tener en cuenta los siguientes temas como: problemática ambiental, social y económica del manejo de biocombustibles). Partiendo de lo dicho anteriormente se elaborara, implementara y validara el modulo desde el objetivo propuesto en la investigación.

Esto conlleva a reconocer la existencia y la validez de diferentes formas de aproximación a los problemas, por parte de los estudiantes, atendiendo a la naturaleza de los mismos y a los intereses de la investigación.

\section{Resultados esperados}

La investigación desarrollada se enfoca desde el campo Experimental - Aplicado buscando el conocer para hacer, para actuar, para construir, y para modificar, en un ambiente determinado; por ello la misma se sustenta desde la elaboración y desarrollo de un modulo de soporte basado en competencias investigativas, el cual recoge todos los aspectos implicados en la investigación como una forma de dar a conocer a los estudiantes en forma didáctica el trabajo de investigación. La estrategia didáctica escogida para desarrollar competencias investigativas en los estudiantes es la resolución de problemas.

Como resultado se espera formar profesionales capaces de desarrollarse en un contexto general a través de habilidades científicas e investigativas, desde un pensamiento critico reflexivo, sistémico, abierto y creativo, que permita la valoración critica de los conocimientos cient́ficos por medio de los trabajos prácticos de laboratorio aplicados en un contexto real.

Al resolver problemas se debe destacar como estos sean significados, comprendidos y abordados y no depender solamente del grado de aprendizaje de una determinada disciplina.

\section{A manera de síntesis}

Las actividades planteadas para el desarrollo de la investigación, permiten que las relaciones enseñanza aprendizaje, sean vistas desde las competencias investigativas como una construcción continua que permiten "una actuación idónea que emerge en una tarea concreta, en un contexto con sentido, donde hay un conocimiento asimilado con propiedad y el cual actúa para ser aplicado en una situación determinada, de manera suficientemente flexible como para proporcionar soluciones variadas y pertinentes (Bogoya, 2000)". De esta manera se hace representativa la participación en la generación, tanto a nivel pedagógico como disciplinar, de nuevas altemativas o proyectos en beneficio de la sociedad. Por ello se hace uso de la producción de biodiesel, utilizando como materia prima la semilla de la Jatropha Curcas a partir de fenómenos catalíticos, como un precedente para desarrollar competencias investigativas en el campo pedagógico, en el campo social y en el campo disaplinar, valiéndonos de la resolución de problemas como una herramienta diferencial en función del contexto de los trabajos prácticos de laboratorio que demandan la solución de un problema, por parte de los estudiantes y la comunidad científica, promoviendo de este modo el interés científico y social, de carácter interdisciplinar, caracterizada por una variedad de significados.

Bibliografía

Bacarat, M.P. y Graziano N. A., (2002). Hacia una cartografía compleja de las Competencias. En S. Tobón, (2004). Formación basada en competencias. Bogotá, Colombia: Editorial Ecoe ediciones. 
Bogoya, D. (2000). Hacia una cartografía compleja de las Competencias. En S. Tobón, (2008). Formación Basada en Competencias, pensamiento complejo, diseño aumiaular y Didáctica. Bogotá, Colombia: Editorial Ecoe ediciones.

Carpenter y Moser (1983). Resolución de Problemas. En F. S Mata, J.L Rodríguez, A. Bolívar. (2004). Diccionario encidopédico de didáctica. (2). Málaga, España: Ediciones Aljibe.

Garcáa, (2001), González, (2000) y Adajian, (1997). Resolución de Problemas. En F. S. Mata, J.L. Rodríguez, A. Bolívar. (2004). Diccionario encidopédico de didáctica. (2). Málaga, España: Ediciones Ajibe.

García R., Oñorbe A., (2006) Resolución de problemas. Alambique didáctica de las ciencias experimentales 48 (abril-junio), 42-49.

Gómez, J. (2001). Competencias: Problemas conceptuales y cognitivos. En S. Tobón, (2008). Formación Basada en Competencias, pensamiento complejo, diseño aumiaular y Didáctica Bogotá, Colombia: Editorial Ecoe ediciones.

Mata F. S., Rodríguez J.L y Bolívar A. (2004). Diccionario encidopédico de didáctica. (2). Málaga, España: Ediciones Ajjibe.

Mendivelso Ojeda M. (2006) Hacia un aprendizaje comprensivo de la investigación. Bogotá, Colombia: IDEP. Bogotá Sin Indiferencia

Tobón, S., (2008). Formación basada en competencias, pensamiento complejo, diseño aurniaular y Didáctica. Bogotá, Colombia: Editorial Ecoe ediciones. 